\title{
COMPLICAÇÕES DE PROCEDIMENTOS LAPAROSCÓPICOS EM UROLOGIA - ANÁLISE RETROPECTIVA DE 1990 A 2002
}

\author{
ANALYSIS OF THE COMPLICATIONS OF LAPAROSCOPIC PROCEDURES IN UROLOGY \\ BETWEEN 1990 AND 2002. A RETROPECTIVE REVIEW
}

\author{
Arakén Almeida' ${ }^{1}$ Lísias Nogueira Castilho²
}

\section{INTRODUÇÃO}

A laparoscopia é uma técnica cirúrgica utilizada com finalidade diagnóstica ou terapêutica em diversas especialidades médicas. Tem como objetivo conseguir, por meio de procedimentos minimamente invasivos, resultados equivalentes ou melhores que aqueles obtidos por procedimentos convencionais ou abertos, levando em consideração as taxas de complicações ${ }^{1}$.

Embora poucas complicações sejam específicas da técnica laparoscópica e a natureza de tais complicações seja semelhante à da cirurgia convencional, sua verdadeira incidência não está claramente determinada ${ }^{1,2}$.

O presente trabalho objetiva descrever e analisar as taxas de complicações de cirurgias laparoscópicas urológicas, conforme as fases da técnica, as características do paciente, a experiência do cirurgião e o tipo de procedimento.

\section{REVISÃO DA LITERATURA}

Utilizando as palavras-chave: complications, laparoscopic e urology, indexadoras de trabalhos citados no INDEX MEDICUS, e suas correspondentes em português, mediante pesquisa bibliográfica, no Medline, no Lilacs e na Biblioteca da Divisão de Clínica Urológica do Hospital das Clínicas da Faculdade de Medicina da Universidade de São Paulo, localizaram-se 262 publicações do período de janeiro de 1990 a junho de 2002. Nessa revisão foram incluídos 167 artigos que compararam complicações de procedimentos abertos e laparoscópicos em Urologia, com mais de 50 cirurgias.

Quanto às complicações relacionadas ao paciente, em virtude das características técnicas da cirurgia laparoscópica, foram considerados separadamente os pacientes pediátricos, os obesos e as gestantes.

Para a avaliação da perícia do cirurgião por meio da curva de aprendizado, consideraram-se 22 trabalhos com um mínimo de 20 pacientes operados.

As complicações foram classificadas em maiores e menores, segundo critérios diversos adotados pelos au- tores, para estimar sua gravidade. As taxas de complicações foram discutidas e analisadas, considerando suas relações com as fases da técnica: acesso, insuflação, dissecção e encerramento; as características do paciente pediátrico, do obeso e da gestante; a experiência do cirurgião e o tipo de procedimento, agrupando-o segundo topografia anatômica.

\section{RESULTADOS}

Foram localizados 167 artigos publicados entre 1990 e 2002, que abordaram as complicações da técnica laparoscópica, relacionadas ao acesso e à insuflação, incluindo um total de 9.683 procedimentos urológicos diversos, com taxas de complicações variando de $0,2 \%$ a 7,5\%. As taxas de complicações referentes a dissecção e ao encerramento foram relatadas em 126 trabalhos, ocorridas em 3.322 procedimentos diversos, variando entre $0,42 \%$ e 8,29\%. No Quadro 1, estão resumidas as complicações das fases da técnica laparoscópica: acesso (introdução da agulha de Veress ou do trocarte), insuflação, dissecção e encerramento.

Em 21 artigos, foram relatados 5.600 casos de diversas cirurgias pediátricas e respectivas complicações, que foram mais freqüentes nas fases de acesso e insuflação, predominantemente em procedimentos renais laparoscópicos transperitoneais. As taxas de complicações variaram entre $0,15 \%$ e $5,4 \%$, sendo a mais freqüente a perfuração peritoneal (Quadro 2).

Dentre 1.036 casos de pacientes obesos, relatados em oito artigos, incluindo linfadenectomias, nefrectomias e colposuspensões, foram mais freqüentes as complicações nas fases de acesso (lesão de nervos periféricos) e insuflação (enfisema subcutâneo). A taxa de complicações variou entre 0,16\% e 29\% (Quadro 2).

Foram localizados quatro artigos sobre cirurgia laparoscópica durante a gestação, descrevendo quatro casos de adrenalectomia para feocromocitoma, operados com sucesso e sem complicações (Quadro 2).

\footnotetext{
1. Professor Assistente da Disciplina de Urologia da Faculdade de Ciências Médicas da Universidade de Pernambuco; Chefe do Serviço de Urologia do Hospital Universitário Oswaldo Cruz da Universidade de Pernambuco.

2. Professor Titular da Pontifícia Universidade Católica de Campinas - São Paulo; Chefe do Serviço de Urologia.
}

Recebido em 07/07/06

Aceito para publicação em 02/09/06

Conflito de interesses: nenhum

Fonte de financiamento: nenhuma

Trabalho realizado na Faculdade de Medicina da Universidade de São Paulo. 
Quanto às complicações dos procedimentos laparoscópicos relacionadas ao cirurgião, 22 artigos, publicados entre 1994 e 2002, preencheram os critérios de inclusão, nos quais foram analisadas 1.421 cirurgias realizadas por profissionais em fase de treinamento. O tempo operatório médio, a taxa média de complicações (variando entre 2,8\% e $14 \%$ ) e a de conversões para cirurgia aberta foram maiores nos 20 a 50 procedimentos iniciais da curva de aprendizagem, reduzindo-se com o aumento do número de casos operados.

No Quadro 3 estão expressos os dados referentes a laparoscopia diagnóstica, orquipexia, orquiectomia e varicocelectomia; no Quadro 4, apresentam-se os dados relativos a colpossupensão vesical, linfocelectomia e linfadenectomia pélvica. O Quadro 5 resume os resultados das prostatectomias radicais; do Quadro 6 constam os dados referentes a nefropexia, marsupialização de cistos renais, neferctomia simples, parcial e de doador vivo, além de nefroureterectomia e pieloplastia. No Quadro 7, expressam-se as informações de adrenalectomias.

\section{COMPLICACÕES DAS FASES DA TÉCNICA LAPAROSCÓPICA}

Abordando-se as complicações quanto às fases da técnica laparoscópica, independente do tipo de paciente e do procedimento, identifica-se que é o acesso a fase de maior risco, devido a inserção do trocarte ou da agulha de Veress serem o aspecto técnico mais perigoso. Oferecem maior risco de lesões vasculares, principalmente dos vasos da parede abdominal e da região intra-abdominal, além das lesões viscerais $^{3}$. Dentre os vasos intra-abdominais, a aorta e a veia

Quadro 1 - Potenciais complicações relatadas em cirurgia laparoscópica de acordo com as fases da técnica.

\begin{tabular}{|c|c|}
\hline Fases da técnica & Complicações relatadas em cirurgia laparoscópica \\
\hline Acesso & $\begin{array}{l}\text { lesões vasculares (vasos da parede abdominal, retroperitoneais e mesentéricos), lesões viscerais (estomago } \\
-7 \% \text {, intestino delgado }-58 \% \text {, cólon }-32 \% \text {, fígado, baço e bexiga }\end{array}$ \\
\hline Insuflação & $\begin{array}{l}\text { enfisema, tensão do pneumoperitônio, pneumotórax, pneumomediastino, embolia gasosa (taxa variando de } \\
0,002 \% \text { a } 0,02 \% \text { ), falha em manter o pneumoperitônio, arritmia cardíaca, hipercapnia, hipotensão }\end{array}$ \\
\hline Dissecção & $\begin{array}{l}\text { lesões térmicas (causa de } 50 \% \text { das lesões viscerais), inabilidade para completar o procedimento, lesões } \\
\text { vasculares (complicações mais freqüentes da fase), lesões viscerais, lesões neurológicas, linfedema/linfocele }\end{array}$ \\
\hline Encerramento & $\begin{array}{l}\text { hemorragia, lesões da parede abdominal, deiscência de ferida, hérnias, dor em ombro (taxa variando de } 5,9 \% \\
\text { a } 6,1 \% \text { ) }\end{array}$ \\
\hline
\end{tabular}

FONTE: Adaptado de Capelouto e Kavoussi (3).

Quadro 2 - Percentuais de complicações relacionadas ao paciente de procedimentos laparoscópicos, segundo trabalhos publicados entre 1990 e 2001.

\begin{tabular}{|c|c|}
\hline Dados & Descrição \\
\hline & Pacientes pediátricos \\
\hline Período & 1996 - 2001, incluídos 21 artigos \\
\hline Procedimento & $\begin{array}{l}5.600 \text { casos, incluídos } 88 \text { procedimentos urológicos, retroperitoneoscópicos e } 62 \text { nefrectomias - } \\
\text { pacientes com idade de } 2 \text { meses a } 16 \text { anos }\end{array}$ \\
\hline Taxa & variando entre $0,15 \%$ e $5,4 \%$ \\
\hline \multirow[t]{3}{*}{ Descrição das complicações } & $\begin{array}{l}\text { Maiores: perfuração peritoneal (a mais freqüente), insuflação pré-peritoneal }(3,2 \%) \text { e enfisema } \\
\text { subcutâneo }(0,94 \%)\end{array}$ \\
\hline & $\begin{array}{l}\text { Menores: lesão vascular }(0,43 \%) \text {, lesão intestinal }(0,17 \%) \text {, lesão vesical }(0,17 \%) \text { e hérnia de } \\
\text { parede abdominal }(0,15 \%)\end{array}$ \\
\hline & Pacientes obesos \\
\hline Período & 1993 - 2003, incluídos 8 artigos \\
\hline Procedimento & 1.036 casos, incluídos 449 linfadenectomias, 249 nefrectomias e 338 colpossuspensões \\
\hline \multirow[t]{2}{*}{ Taxa } & De complicações variando entre $0,16 \%$ e $29 \%$ \\
\hline & De conversão, aproximadamente igual a $12 \%$ \\
\hline \multirow[t]{3}{*}{ Descrição das complicações } & Maiores: enfisema subcutâneo $(3,2 \%)$ e perfuração peritoneal $(0,8 \%)$ \\
\hline & $\begin{array}{l}\text { Menores: lesão de nervos periféricos }(2,4 \%) \text { e lesão por trocarte em órgãos }(1,6 \%) \text { e lesão } \\
\text { vascular }(0,43 \%)\end{array}$ \\
\hline & Gestantes \\
\hline Período & 1997 - 2002, incluídos 4 artigos \\
\hline Procedimento & Quatro adrenalectomias \\
\hline Taxa & Nenhuma complicação relatada \\
\hline
\end{tabular}


Quadro 3 - Resultados de procedimentos de laparoscopia diagnóstica, orquipexia, orquiectomia e varicocelectomia com respectivas complicações, segundo trabalhos publicados entre 1990 e 2001.

\begin{tabular}{|c|c|}
\hline Dados & Descrição \\
\hline \multicolumn{2}{|l|}{ Laparoscopia diagnóstica } \\
\hline Período & 1992 - 2001, incluídos 16 artigos \\
\hline Procedimento & $\begin{array}{l}2.187 \text { cirurgias, compreendendo } 668 \text { criptorquidias, } 419 \text { testículos impalpáveis, } 61 \text { casos de anel } \\
\text { inguinal interno e } 1.100 \text { casos de laparoscopia diagnóstica }\end{array}$ \\
\hline Taxa & $\begin{array}{l}\text { Geral de complicações: } 0,007 \% \text { e } 3,2 \% \\
\text { máxima de } 1,6 \% \text { para criptorquidias, } 3,2 \% \text { para testículos impalpáveis, } 3,75 \% \text { para laparoscopia } \\
\text { diagnóstica }\end{array}$ \\
\hline Descrição das complicações & $\begin{array}{l}\text { Maiores: lesão de sigmóide, lesão vascular, laceração intestinal, aderências intestinais } \\
\text { Menores: da fase de insuflação }\end{array}$ \\
\hline \multicolumn{2}{|l|}{ Orquipexia e orquiectomia } \\
\hline Período & $1999-2000$, incluídos 5 artigos \\
\hline Procedimento & 59 casos, Incluídas 52 orquipexias e 7 orquiectomias \\
\hline Taxa & Máxima de $3.8 \%$, exclusivamente para orquiepexia \\
\hline Descrição das complicações & Maiores: 2 casos de íleo prolongado \\
\hline \multicolumn{2}{|l|}{ Varicocelectomia } \\
\hline Período & 1993 - 2001, incluídos 11 artigos \\
\hline Procedimento & 1.731 casos \\
\hline Taxa & Geral de complicações: $1,3 \%$ e $14,3 \%$ \\
\hline Descrição das complicações & $\begin{array}{l}\text { Maiores: hemorragia, lesão térmica de nervo genito-femural, hematoma pélvico, lesão da veia } \\
\text { epigástrica inferior, lesão de vaso deferente } \\
\text { Menores: granuloma inguinal, enfisema escrotal, hidrocele, infecção de ferida, herniação } \\
\text { intestinal, dor no ombro, hematoma escrotal e outras }\end{array}$ \\
\hline
\end{tabular}

ilíaca comum são as mais lesadas, seguidas dos vasos mesentéricos e da veia cava inferior ${ }^{4}$. As lesões viscerais freqüientemente não eram diagnosticadas no ato operatório ${ }^{1}$. O método preconizado por Hasson, que prescinde da agulha de Veress, reduz a taxa de complicações e de conversão para cirurgia aberta, mas não é completamente isento de riscos, principalmente em pacientes obesos.

$\mathrm{Na}$ fase de insuflação, apesar do aprimoramento da técnica laparoscópica, observou-se que a distensão do espaço pré-peritoneal e o enfisema subcutâneo, conforme a extensão ou a intensidade, ainda persistem como complicações frequientes, podendo produzir pneumotórax, pneumomediastino ou hipercapnia, enquanto que a embolia gasosa, resultante da insuflação do gás nos vasos através da agulha de Veress, é uma complicação extremamente rara ${ }^{5}$.

Na fase de dissecção, as complicações mais freqüentes são as lesões vasculares, estando em segundo lugar as lesões viscerais, das quais $50 \%$ são causadas por lesões térmicas. Ambas constituem um problema importante que pode requerer conversão para cirurgia aberta ${ }^{1,6}$.

$\mathrm{Na}$ fase de encerramento, com a redução da pressão intra-abdominal, alguns vasos de pequeno calibre não sangrantes podem ser focos de sangramento, como relatado em vasos epigástricos, lesados durante a inserção do trocarte. As hérnias incisionais são complicações menores, especialmente em crianças, e ocorrem em orifícios de inserção de trocarte de $10 \mathrm{~mm}$, quando não suturados. Uma complicação freqüente é a dor no ombro. Ela decorre da irritação direta do diafragma pelo $\mathrm{CO}_{2}$ ou por seu subproduto, o ácido carbônico, durante a fase de insuflação, mas não raro manifesta-se no pós-operatório imediato, parecendo independer da abordagem retroperitoneal ou transperitoneal ${ }^{7}$.

\section{COMPLICAÇÕES RELACIONADAS AO PACIENTE}

Considerando as complicações relacionadas ao paciente, a literatura consultada enfatiza crianças, obesos e gestantes como grupos especiais, quer pelas características fisiológicas, quer pelos riscos a que estão expostos, distintamente das demais faixas etárias. A aceitação da técnica em pacientes pediátricos tem gerado polêmica, no que concerne aos custos e benefícios e às potenciais complicações. Nessa população, são bastante semelhantes o tempo de recuperação e a permanência hospitalar dos pacientes operados por via laparoscópica ou aberta, mas, nos 21 artigos analisados, parece haver consenso de que as complicações estão mais relacionadas à experiência do cirurgião e à técnica, do que às características do paciente.

No grupo de pacientes obesos, os autores julgaram que as taxas de complicações intra-operatórias são semelhantes àquelas de pacientes não obesos operados pela mesma técnica, embora haja maior dificuldade de visibilidade e de dissecção dos tecidos pela obesidade excessiva ou por sangramento. A incidência de problemas cardiorrespiratórios é maior no período pós-operatório ${ }^{8}$.

Na literatura urológica, as gestantes são consideradas um grupo a parte. Todavia no presente trabalho, dada a escassez de artigos, admite-se que a utilização desses procedi- 
Quadro 4 - Resultados de procedimentos de colpossupensão vesical, linfocelectomia e linfadenectomia pélvica, com respectivas complicações, segundo trabalhos publicados entre 1990 e 2001.

\begin{tabular}{|c|c|}
\hline Dados & Descrição \\
\hline \multicolumn{2}{|l|}{ Colpossupensão vesical } \\
\hline Período & 1993 - 2001, incluídos 6 artigos \\
\hline Procedimento & 962 casos \\
\hline \multirow[t]{3}{*}{ Taxa } & Geral de complicações: $2,0 \%$ - 15,8\% \\
\hline & De complicações maiores: $3,4 \%$ - 10,0\% \\
\hline & De complicações menores: $5,1 \%$ - $8,8 \%$ \\
\hline \multirow[t]{2}{*}{ Descrição das complicações } & Maiores: lesão térmica de ureter, lesão de bexiga, lesão vascular \\
\hline & $\begin{array}{l}\text { Menores: instabilidade detrussora, dificuldade miccional, infecção urinaria, deiscência deferida, } \\
\text { retenção urinária }\end{array}$ \\
\hline \multicolumn{2}{|l|}{ Linfocelectomia } \\
\hline Período & 1999-2000, incluídos 4 artigos \\
\hline Procedimento & 250 casos \\
\hline Taxa & $0,5 \%-9,0 \%$ \\
\hline Descrição das complicações & $\begin{array}{l}\text { Maiores: lesão de trato urinário (taxa de } 7 \% \text {, em bexiga e ureter transplantado), lesão de cápsula } \\
\text { renal, lesão de artéria epigástrica inferior, sangramento da janela peritoneal } \\
\text { Menores: hérnia no orifício do trocarte e retencão urinária }\end{array}$ \\
\hline \multicolumn{2}{|l|}{ Linfadenectomia pélvica } \\
\hline Período & 1993 -2002, incluídos 8 artigos \\
\hline Procedimento & 1.671 casos \\
\hline \multirow[t]{3}{*}{ Taxa } & Geral de complicações: $3,7 \%$ e $15,0 \%$ \\
\hline & De complicações maiores: $25,5 \%$ \\
\hline & De complicações menores: $74,5 \%$ \\
\hline
\end{tabular}

mentos é uma alternativa, quando medidas terapêuticas conservadoras ou paliativas tenham resultados ineficazes e a solução do problema não possa ser postergada até o final da gestação.

\section{COMPLICACÕES RELACIONADAS AO CIRURGIÃO}

Quanto às complicações relacionadas ao cirurgião, nos artigos selecionados houve consenso quanto à redução das taxas estarem implicitamente relacionadas ao treinamento e à experiência do cirurgião, todavia os autores alertam para o fato de que o treinamento pós-curso é o único fator preditivo dessa taxa de complicações 9 .

Ao longo do tempo, a crescente experiência dos cirurgiões trouxe três consequiências: queda estatisticamente significante do número de lesões viscerais, redução da taxa de complicações, que requeriam conversão nos procedimentos já bem definidos, e mudança na maneira de tratar as complica-

Quadro 5 - Resultados de procedimentos de prostatectomia radical e respectivas complicações, segundo trabalhos publicados entre 1990 e 2001.

Dados Descrição

\section{Prostatectomia radical}

Período

Procedimento

Taxa

Descrição das complicações
2000 - 2002, incluídos 7 artigos

1.480 casos

Geral de complicações: $8,0 \%$ e $35,7 \%$

De complicações maiores: $3,7 \%$ - $4,7 \%$

De complicações menores: $10,4 \%-31,0 \%$

De conversão para cirurgia aberta: $1,2 \%$ a $4,4 \%$

Maiores: necrose tubular aguda, fístula uretro-retal, lesão de reto, lesão de veia ilíaca externa, lesão de alça intestinal

Menores: extravasamento urinário, íleo prolongado, fístula reto-uretral (as três complicações mais freqüentes), retenção urinária, lesão de vasos epigástricos, infecção urinária, tromboflebite, hérnia em local de punção de trocarte, trombose, estenose de ureter, estenose da anastomose vesico-uretral, linforréia, fístula urinária, abscesso de parede abdominal 
Quadro 6 - Resultados de biópsia renal, nefropexia, marsupialização de cisto renal, nefrectomia simples, nefrouretectomia, nefrectomia parcial, nefrectomia de doador vivo, pieloplastia e respectivas complicações, segundo trabalhos publicados entre 1990 e 2001.

\begin{tabular}{ll}
\hline Dados & Descrição \\
\hline Biópsia renal, nefropexia e marsupialização de cisto renal \\
Período & $1994-2001$ incluídos 9 artigos \\
Procedimento & 212 casos \\
Taxa & Geral para biópsia: $8 \%$ \\
& Geral para nefropexia: 2,5\% - 13,0\% \\
& Geral para marsupialização de cisto renal: 3,5\% - 33\% \\
Descrição das complicações & Maiores: urinoma, estenose de ureter \\
& Menores: derrame pulmonar bilateral, retenção urinária, dor local, perfuração peritoneal, \\
& hematoma de retroperitônio, infecção urinária (predominante na nefropexia), extravasamento \\
& urinário (na marsupialização de rim policístico) hipercapnia, enfisema subcutâneo, sangramento \\
& em orifício de trocarte, lesão de diafragma, íleo prolongado, paralisia de nervo fibular
\end{tabular}

\section{Nefrectomia simples}

Período

Procedimento

Taxa

Descrição das complicações

\section{Nefrouretectomia}

Período

Procedimento

Taxa

Descrição das complicações

\section{Nefrectomia parcial}

Período

Procedimento

Taxa

Descrição das complicações
1995 - 2001, incluídos 8 artigos

1.679 casos

Geral de complicações: 6,0\% - 30,2\%

Complicações maiores: 3,0\% - 6,5\%. Complicações menores: 3,0\% - 26,5\%

Conversão para cirurgia aberta: 5,4\% - 10,3\%

Maiores: coleção em retroperitônio, lesão de aorta, lesão de vasos renais, infarto do miocárdio, sangramento, hipercapnia, lesão de pleura, embolia pulmonar, perfuração de colo, hematoma infectado, hematoma de parede abdominal, laceração de baço, pneumotórax

Menores: infecção de ferida, estenoses intestinais, fístula pancreática, hérnia em orifício de inserção do trocarte, febre $\left(38^{\circ} \mathrm{C}\right)$, pequenos hematomas assintomáticos, íleo prolongado, retenção urinária, epididimite, insuficiência cardíaca congestiva, fibrilação atrial, pneumonite, paralisia de nervo braquial, síndrome do compartimento lateral da coxa, confusão mental, trombose de fístula artério-venosa, edema pulmonar

2000, incluídos 3 artigos

134 casos

Geral de complicações: $12,0 \%-48,0 \%$

Complicações maiores: 7,1\% - 8,0\%. Complicações menores: 7,1\% - 10,0\%

Conversão para cirurgia aberta: $4,5 \%-4,8 \%$

Maiores: lesão de veia renal, extravasamento de fluido e suspeita de infiltração do hilo renal Menores: atelectasia

2000 - 2002, incluídos 8 artigos

103 casos

Geral de complicações: $10 \%$ e $12 \%$

Complicações maiores: $6 \%$ - 14,5\%. Complicações menores: $6 \%$

Maiores: lesão vascular, lesão esplênica, lesão de cólon

Menores: extravasamento urinário (aproximadamente $50 \%$ das complicações menores) e atelectasia

1998 - 2002, incluídos 4 artigos

282 casos

Geral de complicações: $12,0 \%$ e 13,0\%

Complicações menores: $11,2 \%$ - 12,5\%.

Conversão para cirurgia aberta: $5,4 \%$ ções, com um significante aumento na proporção de incidentes tratados por laparoscopia ${ }^{10}$. Também concluiu-se que para as nefrectomias laparoscópicas em doenças inflamatórias, as complicações e as conversões para cirurgia aberta dependeram mais da enfermidade do que da experiência do cirurgião ${ }^{11}$. 
Quadro 7 - Resultados de procedimentos de adrenalectomia com respectivas complicações, segundo trabalhos publicados entre 1990 e 2001.

\begin{tabular}{ll}
\hline Dados & Descrição \\
\hline Adrenalectomia & \\
Período & $1997-2001$, incluídos 8 artigos \\
Procedimento & 1.346 casos \\
Taxa & Geral de complicações: $7,7 \%-21,3 \%$ \\
& de complicações maiores: $3,4 \%-8,3 \%$ \\
& de complicações menores: $12,1 \%-12,8 \%$ \\
& de conversão para cirurgia aberta: $0,8 \%-7,0 \%$ \\
Descrição das complicações & Maiores: lesões venosas (principalmente em veias adrenais), lesões viscerais (fígado, pâncreas, \\
& baço) \\
& Menores: atelectasia \\
\hline
\end{tabular}

Ao analisar os trabalhos selecionados, segundo procedimentos urológicos, inicialmente buscou-se identificar a existência de complicações relacionadas ao próprio procedimento. Todavia, embora se tenha podido pontuar algumas observações nesse sentido, ficou evidente que as dificuldades de cada fase da técnica, representam o fator de maior risco de complicações. Algumas constatações pareceram interessantes, podendo ser úteis na execução de procedimentos laparoscópicos urológicos.

\section{COMPLICACCÕES RELACIONADAS AO TIPO DE PROCEDIMENTO}

A laparoscopia diagnóstica para testículos impalpáveis, um procedimento geralmente associado a orquipexia ou orquiectomia, apresenta baixa morbidade e complicações que, mais frequentemente, envolvem o trajeto do trocarte inicial.

Em relação à orquiectomia e à orquidopexia, a maioria dos trabalhos não relata complicações decorrentes desses procedimentos.

$\mathrm{Na}$ varicocelectomia, as complicações são consideradas menores e são mais freqüentes no pós-operatório, predominando os problemas da ferida cirúrgica ${ }^{12}$.

Quanto à colpossuspensão vesical, os autores referem ter havido significante redução da taxa de complicações nos últimos quatro anos, mas não oferecem explicação para a freqüência com que ocorrem a instabilidade do detrusor, e as lesões de bexiga, predominantemente na abordagem transperitoneal.

Os poucos trabalhos identificados sobre linfocelectomia, apresentam séries com reduzido número de casos com baixas taxas de complicações. É importante ressaltar a constatação dos autores de que as lesões iatrogênicas do trato urinário são mais frequientes na cirurgia laparoscópica do que na cirurgia aberta, embora se tenha promovido menor recorrência de linfocele sintomática.

Nas linfadenectomias pélvicas, embora ainda sejam freqüentes a retenção urinária e as lesões vasculares, seguida das lesões de nervos da fossa obturadora e de edema escrotal $^{1,2,5}$ quando comparadas aos procedimentos abertos, os laparoscópicos apresentam menores taxas de complicações.
Quanto à prostatectomia radical, os resultados, inclusive os oncológicos, são semelhantes àqueles do procedimento convencional por via retropúbica, sendo as complicações mais frequentes o extravasamento urinário, as lesões de bexiga, de reto e o íleo prolongado.

Considerando procedimentos como biópsia renal, nefropexia e marsupialização de cisto renal simples, foram relatadas as menores taxas de complicações, podendo-se afirmar serem de fácil execução ${ }^{13}$. No entanto, é na nefrectomia simples, que se identificam as taxas mais elevadas de complicações e conversão para cirurgia aberta, relacionadas aos processos inflamatórios, à litíase e à operação prévia, demonstrando que estas condições ainda permanecem como contraindicações relativas que, podem não permitir a progressão do ato cirúrgico ${ }^{1,3}$.

Vários estudos sobre nefrectomia do doador vivo, destacaram a redução da morbidade no doador e a subseqüente semelhança da função entre rins operados por laparoscopia e pelo método convencional, salientando que as complicações pós-operatórias mais freqüentes foram semelhantes em ambas as técnicas, incluindo a incidência de hérnia e de lesões de nervos do flanco ${ }^{14}$.

Quanto às nefrectomias radical, parcial e nefroureterectomia, há poucos trabalhos na literatura com mais de 50 pacientes. Nos últimos cinco anos, os autores têm enfatizado que a nefrectomia radical não está associada ao aumento do risco de recorrência local, no local de inserção dos trocartes ou à doença metastática ${ }^{15}$. Embora o extravasamento urinário seja uma complicação bastante viável em nefrectomia parcial, as taxas de complicações deste procedimento correspondem aproximadamente à metade daquelas relatadas para o procedimento aberto. Na maioria dos estudos a nefroureterectomia radical equiparou-se à aberta, quanto às complicações, incluídas as oncológicas.

No que concerne a pieloplastia, os artigos relatam complicações intra-operatórias raras, geralmente menores, não relacionadas à abordagem trans ou retroperitoneal nem às técnicas de desmembramento.

$\mathrm{Na}$ adrenalectomia as hemorragias, decorrentes de lesões venosas principalmente de veias adrenais, e à dificuldade de dissecção em virtude de aderências teciduais, são as 
complicações e as causas de conversão mais freqüentes. Os autores alertam que as abordagens transperitoneal e retroperitoneal diferem quanto ao risco e ao tipo de lesões viscerais, sendo quase que exclusivas, respectivamente, para as hepáticas e as pancreáticas ${ }^{16}$.

\section{CONSIDERAÇÕES FINAIS}

Nos últimos 12 anos, a cirurgia urológica laparoscópica tem sido empregada em pacientes pediátricos, obesos e gestantes em procedimentos mais complexos, com taxas reduzidas de complicações, assemelhando-se àquelas correspondentes às cirurgias abertas na dependência das características do procedimento e da experiência do cirurgião. Para utilizar essa técnica, deve-se dedicar especial atenção às fases de acesso e insuflação, nas quais as complicações são menores, mas freqüentemente detectáveis no pós-operatório, e à fase de dissecção, por concentrar as lesões vasculares, que resultam em conversão para a cirurgia aberta na maioria das vezes.

\begin{abstract}
Although laparoscopic surgery is a safe and effective procedure, it is not exempt from risks of complications and death. Complication rates have decreased in various procedures, with means of 1\%, 3.9\% and 9.2\%, for those considered easy, difficult and very difficult, respectively, while death rates have ranged from zero to $0.09 \%$. To analyze the characteristics and the incidence of complications regarding the technique, the patient, the surgeon and the various types of laparoscopic procedures used in urology. A literature review between January 1990 and June 2002 in Medline and Lilacs was undertaken, including approximately 22,000 patients submitted to laparoscopic surgery, classified according to the type of procedure. The complications were considered as major or minor in accordance with various criteria adopted by the authors for appraising their seriousness. The complications regarded as minor ones occurred mainly in the phases of access and insufflation, and were more common in the postoperative period. The ones considered as major were associated with the dissection phase, with more serious characteristics, with vascular lesions predominating over visceral ones. The laparoscopic urological procedures proved to be well tolerated by pediatric and obese patients. Complications rates with this technique were inversely proportional to the experience of the surgeon; they were associated with the complexity of the procedures and were similar to those of the corresponding procedures performed through an open approach. Over ten years, in spite of the increasing complexity of laparoscopic procedures, complications rates have fallen to figures comparable to those of the corresponding open techniques (Rev. Col. Bras. Cir. 2007; 34(1): 54-61).
\end{abstract}

Key words: Urologic surgical procedures/adverse effects; Laparoscopy; Intraoperative complications; Postoperative complications.

\section{REFERÊNCIAS}

1. Vallancien G, Cathelineau X, Baumert H, Doublet JD, Guillonneau B. Complications of transperitoneal laparoscopic surgery in urology: review of 1,311 procedures at a single center. J Urol. 2002;168(1):23-6.

2. Soulié M, Salomon L, Seguin P, Mervant C, Mouly P, Hoznek A, Antiphon P, Plante P, Abbou CC. Multi-institutional study of complications in 1085 laparoscopic urologic procedures. Urology. 2001;58(6):899-903.

3. Capelouto C, Kavoussi LR. Complications of laparoscopic surgery. Urology. 1993;42(1):2-12.

4. Montero M, Tellado MG, Rios J, Mendez R, Somoza I, Pais E, Vela D. Aortic injury during diagnostic pediatric laparoscopy. Surg Endosc. 2001;15(5):519. Epub 2001 Mar 13.

5. Fahlenkamp D, Rassweiler F, Fornara P, Frede T, Loening SA. Complications of laparoscopic procedures in urology: experience with 2,407 procedures at 4 german centers. J Urol. 1999;162(3 Pt 1):765-71.

6. Parra RO, Hagood PG, Boullier JA, Cummings JM, Mehan DJ. Complications of laparoscopic urological surgery: experience at St. Louis University. J Urol. 1994;151(3):681-4.

7. Rassweiler J, Frede T, Henkel TO, Stock C, Alken P. Nephrectomy: a comparative study between the transperitoneal and retroperitoneal laparoscopy versus the open approach. Eur Urol. 1998;33(5):489-96.
8. Jacobs SC, Cho E, Dunkin BJ, Bartlett ST, Flowers JL, Jarrell B, Jacobs SC. Laparoscopic nephrectomy in the markedly obese living renal donor. Urology. 2000;56(6):926-9.

9. See WA, Cooper CS, Fisher RJ. Predictor of laparoscopic complications after formal training in laparoscopy surgery. JAMA. 1993;270(22):2689-92.

10. Chapron C, Querleu D, Mage G, Madelenat P, Dubisson JB, Audebet A, Erny R, Bruhat MA. Complications de la coeliochirurgie gynecologique: etude multicentrique a partir de 7604 celioscopies. J Gynecol Obstet Biol Reprod. 1992;21:20713.

11. Keeley FX, Tolley DA. A review of our first 100 cases of laparoscopic nephrectomy: defining risk factors for complications. Br J Urol. 1998;82(5):615-8.

12. Podkamenev YVV, Stalmakhovich VN, Urkov PS, Solovjev AA, Iljin VP. Laparoscopic surgery for pediatric varicoceles: randomized controlled trial. J Pediatr Surg. 2002;37(5):727-9.

13. Plas E, Daha K, Riedl C, Hübner W, Pflüger H. Long-term followup after laparoscopic nephropexy for symptomatic nephroptosis. J Urol. 2001;166(2):449-52.

14. Brown SL, Biehl TR, Rawlins MC, Hefty TR. Laparoscopic live donor nephrectomy: a comparison with the conventional approach. J Urol. 2001;165(3):766-9.

15. Ono Y, Kinukawa T, Hattori R, Gotoh M, Kamihrira O, Ohshima $\mathrm{S}$. The long-term outcome of laparoscopic radical nephrectomy for small renal cell carcinoma. J Urol. 2001;165(6 Pt 1):1867-70. 
16. Terachi T, Yoshida O, Matsuda T, Orikasa S, Chiba Y, Takahashi K, Takeda M, Higashihara E, Murai M, Baba S, Fujita K, Suzuki K, Ohshima S, Ono Y, Kumazawa J, Naito S. Complications of laparoscopic and retroperitoneoscopic adrenalectomies in 370 cases in Japan: a multi-institutional study. Biomed Pharmacother. 2000;54 Suppl 1:S211-4.

Como citar este artigo:

Araújo AA. Complicações de procedimentos laparoscópicos em urologia - análise retrospectiva de 1990 a 2002. Artigo de revisão. Rev Col Bras Cir. [periódico na Internet] 2007;34(1). Disponível em URL: http://www.scielo.br/rcbc
Endereço para correspondência:

Rua Clóvis de Castro, 250 apto 701

Parnamirim

52060-180 - Recife - PE

Fone (81) 3441-9095

Fax: (81) 3221-2840

E-mail: arakenaa@elogica.com.br 